\title{
Caracterización del servicio de alojamiento turístico usando métodos multivariados en el cantón Tena, provincia Napo
}

\author{
Characterization of the Tourist Accommodation Service Using Multivariate \\ Method in the Tena Canton, Napo Province
}

\author{
Jaimar Andrea Cifuentes Caiza. ${ }^{1}$, Edison Oliver Segura Chávez. ${ }^{2}$, Olger Eugenio \\ Gavilánez Pacheco. ${ }^{3}$ \& Cintia del Pilar Hernández Espinosa. ${ }^{4}$
}

\begin{abstract}
.
DOI: https://doi.org/10.33262/concienciadigital.v4i2.2.1780

The present research work was carried out in the Tena canton, Napo province in order to characterize the tourist accommodation service, with cut-off date October 2019, by using the multivariate statistical method. During the research process, 92 establishments included in the tourist registry were considered as study subjects, which were subjected to a KMO sufficiency analysis using 30 variables. By means of statistical analysis, the variables were grouped into six components for the interpretation of the statistical system in a percentage equivalent to $57.03 \%$. The information collection instrument was validated using the Cronbach's Alpha method, giving a reliability of 0.873 . The tourist accommodation service was developed under empirical bases from the perspective of its administrators with a national tourist flow. It is important to innovate without forgetting that tourist activities are characterized by being dynamic and highly seasonal.
\end{abstract}

Keywords: tourism accommodation, inversion, multivariate method.

\footnotetext{
${ }^{1}$ Universidad Estatal Amazónica, Maestrante del Centro de Posgrado, Pastaza, Ecuador. ja.cifuentesc@uea.edu.ec, https://orcid.org/0000-0001-7160-4723

2 Universidad Estatal Amazónica, Facultad de Ciencias de la Vida, Pastaza, Ecuador, esegura@uea.edu.ec, https://orcid.org/0000-0002-5274-8825

${ }^{3}$ Ministerio de Turismo del Ecuador, Ingeniero en ecoturismo, Magister en ecoturismo y Manejo de Áreas Naturales, gavilanez88@ gmail.com, https://orcid.org/0000-0002-5030-5895

${ }^{4}$ Empresa Pública IkiamEp., Unidad de Proyectos, Tena, Ecuador. cynthiaphe77@hotmail.com, https://orcid.org/0000-0002-1118-1332
} 


\section{Resumen.}

El presente trabajo de investigación se realizó en el cantón Tena, provincia Napo con la finalidad de caracterizar al servicio de alojamiento turístico, con fecha corte octubre 2019, mediante la utilización del método estadístico multivariado. Durante el proceso de investigación se consideró como sujetos de estudio a 92 establecimientos que constan en el catastro turístico, mismos que fueron sometidos a un análisis de suficiencia KMO con la utilización de 30 variables. Mediante el análisis estadístico se agrupó las variables en seis componentes para la interpretación del sistema estadístico en un porcentaje equivalente al 57,03\%. El instrumento de recopilación de información fue validado mediante el método de Alfa de Cronbach, dando una confiabilidad del 0,873. El servicio de alojamiento turístico se desarrolló bajo bases empíricas desde la perspectiva de sus administradores con un flujo turístico nacional. Es importante innovar sin olvidar que las actividades turísticas están caracterizadas por ser dinámicas y altamente estacional.

Palabras claves: alojamiento turístico, inversión, método multivariado.

\section{Introducción.}

El turismo se ha convertido en uno de los sectores económicos que aporta significativamente a la consecución de los objetivos de desarrollo convirtiéndose en el tercer sector predominante del comercio internacional, con el $10.4 \%$ del producto interno bruto (PIB), lo que ha permitido generar a nivel mundial 313 millones fuentes de trabajo (Organizacion Mundial del Turismo, 2018).

De acuerdo a la publicación realizada por el Ministerio del Turismo a través de su portal, en el año 2018, este ente rector viabilizó la posibilidad de contar con un portafolio de potenciales inversores en el área de alojamiento, logrando consolidar datos relevantes de 37 proyectos plenamente identificados que facilitan información a los potenciales inversionistas. Para el ente rector de la actividad turística la aplicación y desarrollo de estrategias de promoción de forma interna y externa significó al país una inversión 150 millones de dólares. Adicionalmente, es importante recalcar que, en el año 2018, la llegada de extranjeros al país fue de 2427.600 , existió una contribución directa del 2\% del turismo al PIB del Ecuador, el ingreso de divisas estimadas por turismo receptor resultó ser de \$1.043,4. Se registró 134.774 fuentes de empleo en el sector turístico según el catastro nacional provisional (Ministerio de Turismo, 2018).

Así como también, Ecuador se encuentra entre los países con mejor desempeño económico de Latinoamérica gracias al apoyo para la inversión productiva privada que permite la generación de empleo de calidad y la reducción en la desigualdad de ingresos. Ecuador reporta un 0,27\% de tasa de inflación al mes de diciembre 2018, registrándose como la menor de los países Sur-americanos según datos del Banco Central de este país. Además, el índice de Gini que mide la desigualdad en los ingresos ha permitido determinar que la misma se ha reducido pasando de 0.551 en 2007 a 0.452 en 2018. El 
sector turístico genera un impacto positivo cuando propicia la creación de microempresas que a la vez se convierten en generadores de diversificando la economía local (PRO ECUADOR, 2019).

Mediante el análisis de la inversión en el servicio de alojamiento turístico en el cantón Tena utilizando el método estadístico multivariado se pretende contar con información relevante que permita mejorar la toma de decisiones enmarcadas en la realidad del cantón en un contexto socioeconómico.

\section{Metodología.}

El estudio se realizó en la capital de la provincia de Napo, ubicado en el centro occidente de la Región Amazónica ecuatoriana. Representa el 31.3\% del territorio de la provincia de Napo, esto equivale a una extensión territorial de 3897.41 km2. Ver Ilustración 1.
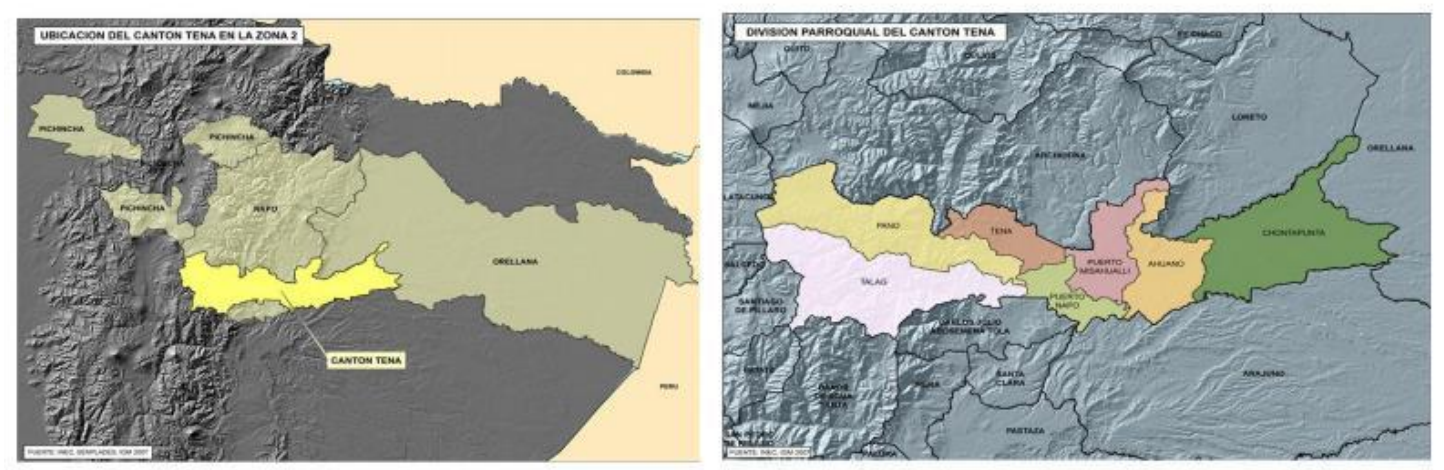

Ilustración 1. Macro y Micro Localización del Cantón Tena

Fuente: SENPLADES (2011)

La investigación se realizó con los administradores y/o propietarios de los 92 establecimientos turísticos evidenciados en la Tabla 2 del cantón Tena con fecha corte octubre 2019.

\section{Tabla 1}

Clasificación de Actividades Turísticas Registradas en el Catastro Turístico del Ministerio de Turismo.

CLASIFICACIÓN DE ACTIVIDADES TURÍSTICAS REGISTRADAS EN EL CATASTRO TURÍSTICO DEL MINISTERIO DE TURISMO

\begin{tabular}{ll}
\hline Número & Actividades Turísticas \\
116 & Alimentos y bebidas \\
92 & Alojamiento \\
6 & Centros de turismo comunitario \\
37 & Operación e intermediación \\
1 & Parques y atracciones estables
\end{tabular}


CLASIFICACIÓN DE ACTIVIDADES TURÍSTICAS REGISTRADAS EN EL CATASTRO TURÍSTICO DEL MINISTERIO DE TURISMO

\begin{tabular}{ll}
\hline Número & Actividades Turísticas \\
2 & Transporte turístico
\end{tabular}

Fuente: Elaboración propia a partir de datos obtenidos del Catastro Turístico MINTUR (2019)

El instrumento de recopilación de información se estructuró con preguntas cerradas. Esta información permitió caracterizar el servicio de alojamiento existente en el cantón Tena y determinar la demanda actual identificada por parte de los administradores y/o propietarios de los establecimientos de la actividad turística en estudio. La encuesta fue validada mediante el método de Alpha de Cronbach a través del uso del procesador estadísticos computacional, IBM SPSS Statistics 22. Algoritmos Chicago: IBM SPSS Inc. Dando como resultado que el instrumento tiene un alto grado de fiabilidad del 0,873, según el análisis de todos los ítems.

Para esta investigación se realizó un análisis estadístico multivariado tomando en cuenta a los 92 sujetos de estudio con 30 variables, de las cuales 3 de ellas no presentaron variabilidad porque su representación fue 0. En la Tabla 2, se enlista las variables definidas para establecer la correlación.

Tabla 2

Varianza de los ítems

\begin{tabular}{llrr}
\hline \multicolumn{3}{c}{ VARIANZA DE LOS ÍTEMS } \\
\hline VAR1 & Grado de Escolaridad del empresario & N & Varianza \\
VAR2 & Profesión del empresario & 92 & 0,492 \\
VAR3 & Edad del empresario & 92 & 0,373 \\
VAR4 & Género del empresario & 92 & 0,062 \\
VAR5 & Procedencia del empresario & 92 & 0,252 \\
VAR6 & Conocimiento del Sistema turístico & 92 & 0,802 \\
VAR7 & Tipo de establecimiento & 92 & 0,200 \\
VAR8 & Etapa de la empresa & 92 & 0,928 \\
VAR9 & Plaza del establecimiento & 92 & 0,287 \\
VAR10 & Precio de la habitación & 92 & 0,843 \\
VAR11 & Categoría del establecimiento & 92 & 0,000 \\
VAR12 & Ventas anuales del establecimiento & 92 & 1,292 \\
VAR13 & Número de empleados & 92 & 0,145 \\
VAR14 & Razón social & 92 & 0,146 \\
VAR15 & Manejo de contabilidad & 92 & 0,130 \\
VAR16 & Cómo dispone de las utilidades & 92 & 0,501 \\
VAR17 & Porcentaje de huéspedes que llegan por & 92 & 0,098 \\
& medio de Agencias de viajes & 92 & 0,865 \\
\hline VAR18 & Capital invertido en la empresa & 92 & 0,670 \\
VAR19 & Actualmente, posee capacidad de inversión & 92 & 0,000 \\
VAR20 & Acudió a un crédito el empresario & 0,172
\end{tabular}




\begin{tabular}{llll} 
VAR21 & Tipo de crédito & 92 & 0,000 \\
VAR22 & Qué mejoraría & 92 & 1,145 \\
VAR23 & Tiempo de resultados que espera en la & 92 & 0,426 \\
& empresa & 92 & 0,796 \\
VAR24 & Factor para toma de decisiones & 92 & 0,702 \\
VAR25 & Rango de edad del cliente & 92 & 0,226 \\
VAR26 & Procedencia del cliente & 92 & 0,428 \\
VAR27 & Número de retorno de cliente & 92 & 0,325 \\
VAR28 & Modalidad de viaje del cliente & 92 & 0,847 \\
VAR29 & Tipo de habitación que solicita el cliente & & 0,128 \\
& frecuentemente & 92 & \\
VAR30 & Motivación de viaje del cliente & 92 & \\
& N válido (por lista) & & \\
\hline
\end{tabular}

Fuente: Elaboración propio con datos obtenidos con el procesador estadístico computacional, IBM SPSS Statistics 22. Algoritmos Chicago: IBM SPSS Inc.

En este caso se realizó un análisis descriptivo para encontrar la media, la desviación estándar, y el coeficiente de variación y así poder comparar la representatividad de la media de todas las variables consideradas.

Con la organización de la información en la base de datos se procedió a realizar la prueba de Bartlett para evidenciar la correlación entre las variables. Para localizar el sistema estadístico se calculó correlaciones bivariadas entre par de variables y formar una matriz con todas las relaciones. El análisis de suficiencia general o Kaiser - Meyer - Olklin resultó ser de 0,71 , dando continuidad al análisis de 6 componentes principales.

\section{Resultados.}

De los 92 sujetos de estudio se identificó la siguiente tipificación de establecimientos.

El análisis estadístico multivariado permitió reducir la dimensión de las 30 variables originando otras que están interrelacionadas a través de componentes principales. Para establecer los componentes principales debe existir variabilidad, como se visualiza en la Tabla 2, es por ello que una vez obtenida la base de datos sobre los estadísticos descriptivos se estableció que tres variables tenían desviación estándar de cero, mismas que fueron omitidas para el estudio.

Tabla 3

Variables con Nivel de Significancia Representativo para el Estudio VARIABLES CON NIVEL DE SIGNIFICANCIA REPRESENTATIVO PARA EL ESTUDIO

\begin{tabular}{lrrr}
\hline Variables & & \multicolumn{2}{l}{$\begin{array}{l}\text { Desviación } \\
\text { N De }\end{array}$} \\
\hline Grado de Escolaridad del empresario & 2,2391 & 0,70117 & 92 \\
\hline Profesional del empresario & 2,0217 & 0,61086 & 92 \\
Edad del empresario & 2,9348 & 0,24826 & 92 \\
Género del empresario & 1,5326 & 0,50167 & 92 \\
Procedencia del empresario & 2,9891 & 0,89559 & 92
\end{tabular}


VARIABLES CON NIVEL DE SIGNIFICANCIA REPRESENTATIVO PARA EL ESTUDIO

\begin{tabular}{lrrr}
\hline & & Desviación & \multicolumn{2}{l}{ N De } \\
Variables & Media & Estándar & Análisis \\
\hline Conoce del Sistema turístico & 1,7283 & 0,44729 & 92 \\
Tipo de establecimiento & 3,5761 & 0,96344 & 92 \\
Etapa de la empresa & 2,9022 & 0,53575 & 92 \\
Plaza del establecimiento & 1,7609 & 0,91831 & 92 \\
Categoría del establecimiento & 4,0652 & 1,13684 & 92 \\
Ventas anuales del establecimiento & 1,1739 & 0,38111 & 92 \\
Número de empleados & 1,087 & 0,38236 & 92 \\
Razón social del establecimiento & 1,1522 & 0,36116 & 92 \\
Manejo de contabilidad & 2,0652 & 0,70795 & 92 \\
Cómo dispone de las utilidades & 2,0326 & 0,31277 & 92 \\
Porcentaje de huéspedes que llegan por medio de & & & \\
Agencias de viajes & 1,5543 & 0,93013 & 92 \\
Capital invertido en la empresa & 2,5109 & 0,81866 & 92 \\
Acudió a un crédito & 1,7826 & 0,41473 & 92 \\
Qué mejoraría al reinvertir & 2,2283 & 1,07011 & 92 \\
Tiempo de resultados que espera en la empresa & 1,9457 & 0,65237 & 92 \\
Factor para toma de decisiones & 2,0761 & 0,89238 & 92 \\
Rango de edad del cliente & 4,6087 & 0,83806 & 92 \\
Procedencia del cliente & 1,337 & 0,47526 & 92 \\
Número de retorno de cliente & 1,9891 & 0,65456 & 92 \\
Modalidad de viaje del cliente & 2,9348 & 0,57041 & 92 \\
Tipo de habitación que solicita el cliente frecuentemente & 2,7935 & 0,92032 & 92 \\
Motivación de viaje del cliente & 1,1196 & 0,35834 & 92 \\
\hline Fute: Elat & & & \\
\hline
\end{tabular}

Fuente: Elaboración propio con datos obtenidos con el procesador estadístico computacional, IBM SPSS Statistics 22. Algoritmos Chicago: IBM SPSS Inc.

En la Tabla 4, se estima que con el 57,03\% de varianza el sistema puede ser explicado, por lo tanto, para el presente estudio se establece 6 componentes principales.

\section{Tabla 4}

Varianza de las Variables Explicadas

\begin{tabular}{|c|c|c|c|c|c|c|c|c|c|}
\hline \multicolumn{10}{|c|}{ VARIANZA DE LAS VARIABLES EXPLICADAS } \\
\hline \multirow{2}{*}{ Componente } & \multicolumn{3}{|c|}{ Autovalores Iniciales } & \multicolumn{3}{|c|}{$\begin{array}{c}\text { Sumas de Extracción de } \\
\text { Cargas al Cuadrado }\end{array}$} & \multicolumn{3}{|c|}{$\begin{array}{c}\text { Sumas de Rotación de Cargas } \\
\text { al Cuadrado }\end{array}$} \\
\hline & $\underline{\text { Total }}$ & $\begin{array}{c}\frac{\% \text { de }}{\text { Varianza }} \\
\end{array}$ & $\frac{\%}{\underline{\text { Acumulado }}}$ & Total & $\begin{array}{c}\frac{\% \text { de }}{\text { Varianza }} \\
\end{array}$ & $\begin{array}{c}\underline{\%} \\
\underline{\text { Acumulado }}\end{array}$ & Total & $\begin{array}{c}\frac{\% \text { de }}{\text { Varianza }} \\
\end{array}$ & $\begin{array}{c}\frac{\%}{\text { Acumulado }} \\
\underline{\text { Acula }}\end{array}$ \\
\hline 1 & 6,163 & 22,826 & 22,826 & 6,163 & 22,826 & 22,826 & 5,330 & 19,743 & 19,743 \\
\hline 2 & 2,439 & 9,035 & 31,861 & 2,439 & 9,035 & 31,861 & 2,456 & 9,097 & 28,840 \\
\hline 3 & 2,204 & 8,164 & 40,025 & 2,204 & 8,164 & 40,025 & 2,273 & 8,417 & 37,257 \\
\hline 4 & 1,650 & 6,113 & 46,138 & 1,650 & 6,113 & 46,138 & 1,895 & 7,017 & 44,274 \\
\hline 5 & 1,528 & 5,660 & 51,798 & 1,528 & 5,660 & 51,798 & 1,823 & 6,754 & 51,027 \\
\hline 6 & 1,411 & 5,227 & 57,025 & 1,411 & 5,227 & 57,025 & 1,619 & 5,997 & 57,025 \\
\hline 7 & 1,270 & 4,703 & 61,728 & & & & & & \\
\hline 8 & 1,118 & 4,140 & 65,867 & & & & & & \\
\hline
\end{tabular}


ISSN: 2600-5859

\begin{tabular}{|c|c|c|c|c|c|c|c|c|c|}
\hline \multicolumn{10}{|c|}{ VARIANZA DE LAS VARIABLES EXPLICADAS } \\
\hline \multirow{2}{*}{ Componente } & \multicolumn{3}{|c|}{ Autovalores Iniciales } & \multicolumn{3}{|c|}{$\begin{array}{l}\text { Sumas de Extracción de } \\
\text { Cargas al Cuadrado }\end{array}$} & \multicolumn{3}{|c|}{$\begin{array}{c}\text { Sumas de Rotación de Cargas } \\
\text { al Cuadrado }\end{array}$} \\
\hline & $\underline{\text { Total }}$ & $\frac{\% \text { de }}{\underline{\text { Varianza }}}$ & $\begin{array}{c}\underline{\%} \\
\underline{\text { Acumulado }}\end{array}$ & $\underline{\text { Total }}$ & $\frac{\% \text { de }}{\text { Varianza }}$ & $\begin{array}{c}\frac{\%}{2} \\
\text { Acumulado }\end{array}$ & $\underline{\text { Total }}$ & $\frac{\% \text { de }}{\text { Varianza }}$ & $\begin{array}{c}\frac{\%}{\underline{O}} \\
\text { Acumulado }\end{array}$ \\
\hline 9 & 1,082 & 4,008 & 69,875 & & & & & & \\
\hline 10 & 0,929 & 3,440 & 73,316 & & & & & & \\
\hline 11 & 0,821 & 3,041 & 76,356 & & & & & & \\
\hline 12 & 0,786 & 2,911 & 79,268 & & & & & & \\
\hline 13 & 0,660 & 2,444 & 81,711 & & & & & & \\
\hline 14 & 0,636 & 2,356 & 84,068 & & & & & & \\
\hline 15 & 0,574 & 2,127 & 86,195 & & & & & & \\
\hline 16 & 0,540 & 2,001 & 88,196 & & & & & & \\
\hline 17 & 0,483 & 1,787 & 89,983 & & & & & & \\
\hline 18 & 0,428 & 1,585 & 91,568 & & & & & & \\
\hline 19 & 0,378 & 1,399 & 92,967 & & & & & & \\
\hline 20 & 0,312 & 1,156 & 94,123 & & & & & & \\
\hline 21 & 0,299 & 1,109 & 95,232 & & & & & & \\
\hline 22 & 0,286 & 1,061 & 96,293 & & & & & & \\
\hline 23 & 0,248 & 0,917 & 97,209 & & & & & & \\
\hline 24 & 0,238 & 0,882 & 98,091 & & & & & & \\
\hline 25 & 0,203 & 0,753 & 98,844 & & & & & & \\
\hline 26 & 0,191 & 0,709 & 99,553 & & & & & & \\
\hline 27 & 0,121 & 0,447 & 100,000 & & & & & & \\
\hline
\end{tabular}

Fuente: Datos obtenidos con el procesador estadístico computacional, IBM SPSS Statistics 22. Algoritmos Chicago: IBM SPSS Inc.

Posteriormente, se procede a presentar la gráfica de sedimentación donde evidentemente se observa los 6 componentes que conforman el sistema estadístico a analizar.

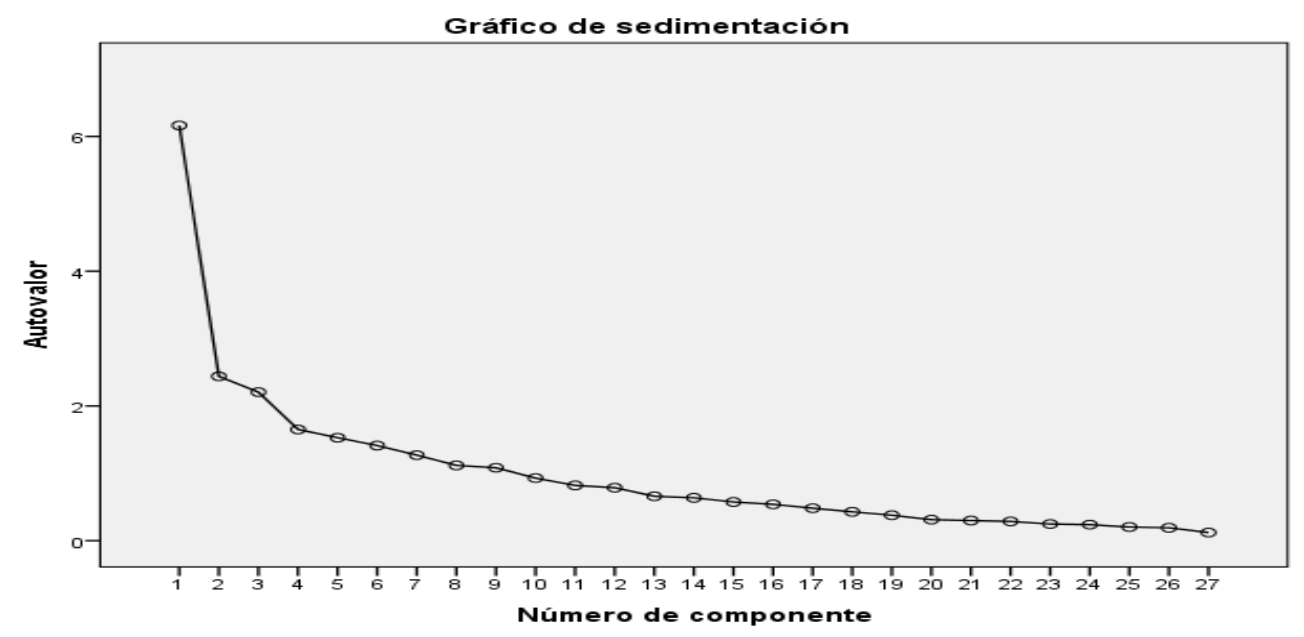

Figura 1. Grafica de Sedimentación del Sistema Estadístico

Fuente: Datos obtenidos con el procesador estadístico computacional, IBM SPSS Statistics 22.

Algoritmos Chicago: IBM SPSS Inc. 
En la Figura 1, se evidencia que a partir del componente 7 el nivel de significancia empieza a disminuir presentando una leve varianza. Por lo tanto, se valida el análisis mediante los 6 componentes propuestos en la Tabla 4.

\section{Tabla 5}

Varianza de Componentes

\begin{tabular}{|c|c|c|c|c|c|c|}
\hline \multicolumn{7}{|c|}{ VARIANZA DE COMPONENTES } \\
\hline \multirow{2}{*}{ VARIABLES } & \multicolumn{6}{|c|}{ COMPONENTES } \\
\hline & 1 & 2 & 3 & 4 & 5 & 6 \\
\hline Grado de Escolaridad & $\mathbf{0 , 5 2 0}$ & 0,257 & 0,424 & $-0,188$ & 0,122 & 0,004 \\
\hline Profesional & $-0,303$ & 0,041 & 0,192 & $-0,071$ & $\mathbf{0 , 5 7 3}$ & 0,002 \\
\hline Edad & $-0,057$ & 0,017 & 0,074 & 0,314 & 0,440 & 0,257 \\
\hline Género & 0,128 & 0,180 & $-0,425$ & 0,329 & 0,039 & $-0,039$ \\
\hline Procedencia & 0,046 & 0,464 & $-0,200$ & 0,118 & 0,543 & $-0,057$ \\
\hline Sistema turístico & $-0,781$ & $-0,071$ & 0,130 & $-0,009$ & 0,186 & $-0,183$ \\
\hline Tipo de establecimiento & $\mathbf{0 , 5 2 7}$ & $-0,179$ & $-0,102$ & 0,127 & 0,284 & $-0,351$ \\
\hline Etapa de la empresa & $-0,102$ & 0,243 & 0,598 & $-0,055$ & 0,316 & 0,179 \\
\hline Plaza del establecimiento & 0,338 & $-0,367$ & 0,354 & 0,044 & 0,154 & $-0,247$ \\
\hline Categoría del establecimiento & $-0,568$ & 0,042 & $-0,027$ & $-0,380$ & $-0,225$ & 0,323 \\
\hline $\begin{array}{l}\text { Ventas anuales del } \\
\text { establecimiento }\end{array}$ & 0,674 & $-0,043$ & 0,023 & 0,275 & $-0,004$ & 0,226 \\
\hline Número de empleados & 0,469 & $-0,300$ & 0,120 & 0,096 & $-0,070$ & $-0,209$ \\
\hline Razón social & 0,383 & $-0,114$ & 0,205 & 0,150 & $-0,221$ & 0,481 \\
\hline Manejo de contabilidad & $-0,794$ & $-0,082$ & 0,028 & 0,080 & 0,084 & $-0,006$ \\
\hline Cómo dispone de las utilidades & 0,001 & 0,590 & 0,464 & $-0,024$ & $-0,210$ & $-0,348$ \\
\hline Porcentaje de huéspedes que & & & & & & \\
\hline $\begin{array}{l}\text { llegan por medio de Agencias de } \\
\text { viajes }\end{array}$ & 0,462 & $\mathbf{0 , 4 7 0}$ & 0,361 & 0,148 & $-0,180$ & $-0,079$ \\
\hline Capital invertido en la empresa & 0,709 & $-0,238$ & 0,014 & 0,020 & 0,321 & 0,123 \\
\hline Acudió a un crédito & $-0,609$ & 0,192 & $-0,167$ & 0,143 & 0,000 & $-0,093$ \\
\hline Qué mejoraría & $\mathbf{0 , 5 8 1}$ & 0,455 & 0,002 & $-0,116$ & $-0,175$ & $-0,150$ \\
\hline $\begin{array}{l}\text { Tiempo de resultados que espera } \\
\text { en la empresa }\end{array}$ & 0,765 & $-0,137$ & 0,049 & $-0,120$ & 0,177 & 0,147 \\
\hline Factor para toma de decisiones & 0,006 & 0,176 & 0,046 & $-0,503$ & 0,147 & 0,518 \\
\hline Rango de edad del cliente & 0,679 & $-0,147$ & 0,008 & $-0,197$ & $-0,132$ & 0,124 \\
\hline Procedencia del cliente & 0,130 & 0,487 & $-0,607$ & 0,156 & $-0,005$ & 0,212 \\
\hline Número de retorno de cliente & $\mathbf{0 , 5 7 7}$ & 0,243 & $-0,306$ & 0,224 & $-0,050$ & $-0,011$ \\
\hline Modalidad de viaje del cliente & $-0,163$ & $-0,666$ & 0,088 & 0,397 & $-0,116$ & 0,147 \\
\hline $\begin{array}{l}\text { Tipo de habitación que solicita el } \\
\text { cliente frecuentemente }\end{array}$ & 0,270 & $-0,299$ & $-0,156$ & $-0,553$ & 0,007 & $-0,327$ \\
\hline Motivación de viaje del cliente & $-0,267$ & 0,111 & 0,567 & 0,456 & $-0,220$ & 0,091 \\
\hline
\end{tabular}

Fuente: Datos obtenidos con el procesador estadístico computacional, IBM SPSS Statistics 22. Algoritmos Chicago: IBM SPSS Inc.

Según la Tabla 5, se presentan las variables que tuvieron valores de pesos superiores a 0,5, suficiente para identificar variables en cada componente seleccionada. según (Hair, Black, Babin, \& Anderson, 2016). 
En la Tabla 4 se explica que el sistema estadístico para esta investigación se confirma por 6 componentes, cada uno conformado por un grupo de variables, como se detalla a continuación:

1. Componente 1: Grado de Escolaridad del empresario, Desconocimiento del Sistema turístico, Tipo de establecimiento, Categoría del establecimiento, Ventas anuales del establecimiento, Número de empleados, Manejo de contabilidad, Capital invertido en la empresa, Acudió a un crédito para invertir, Qué mejoraría al momento de reinvertir, Tiempo de resultados que espera en la empresa, Rango de edad del cliente, Número de retorno de cliente.

Según los datos obtenidos, se puede evidenciar que entre los grados de instrucción Bachiller y Superior poseen una diferencia del 13\% siendo los de mayor ponderación. Mientras que el $14 \%$ de los inversionistas poseen un nivel de instrucción primaria y finalmente el $1 \%$ corresponde a los que no han tenido acceso alguno a la educación formal. El sistema turístico es tema esencial para la gestión del turismo, pero se evidencia que en el cantón Tena el $73 \%$ desconoce sobre la existencia y el $27 \%$ está informado sobre este tema.

Además, el 57\% de establecimientos turísticos que ofertan el servicio de alojamiento son hostales, el $24 \%$ son hosterías, el $12 \%$ hoteles y $7 \%$ entre lodge, casa de huéspedes, resort y campamento turístico. Por la característica de la zona de estudio es más evidente que los inversionistas prefieren implementar sus edificaciones en lugares que permita mayor contacto con la naturaleza.

La categoría de los establecimientos de alojamiento turística con mayor ponderación es de 3 estrellas, quiere decir, que el servicio de alojamiento cumple con un número considerable de requisitos obligatorios exigidos por el ente rector plasmado en la documentación pública (Ministerio De Turismo, 2016). La categoría varía según la tipología del establecimiento. El 4\% de establecimiento tiene categoría Única a la cual pertenecen los campamentos turísticos y casa de huéspedes existentes en el cantón.

Se logró determinar el tipo de empresa según su tamaño por el volumen de ventas anuales, siendo el $83 \%$ pequeñas empresas y el $16 \%$ microempresas. La variable número de empleados tiene relación con la variable ventas anuales, permite validar la confiabilidad de la respuesta para determinar el tamaño de la empresa, pero al determinar que el $93 \%$ de las empresas tienen de 1 a 9 personas, según la Superintendencia de compañías, este número es para microempresas siendo el mayor porcentaje en la Figura 6 las pequeñas empresas, el $5 \%$ prácticamente reflejan pertenecer a pequeñas empresas según su número y el $1 \%$ a medianas empresas tipo B.

Estos datos son muy interesantes ya que refleja la realidad local, tomando en cuenta que muchos de los empresarios que invierten en alojamiento turístico trabaja con un número pequeño de integrantes y/o empleados siendo muchos de ellos familiares, como muchos de ellos manifestaron como argumentación a su respuesta extraoficialmente. 
En cuanto al manejo de contabilidad en las empresas de alojamiento turístico, según datos proporcionados por los inversionistas el 50\% de los involucrados manejan medianamente temas de contabilidad en sus establecimientos, el $28 \%$ ha decidido no aplicar estrategias de contabilidad por falta de tiempo y de interés, mientras que el $22 \%$ lo realiza rigurosamente para garantizar un control de ingresos y egresos, este último resultado se puede contrarrestar con el $71 \%$ de establecimientos que ha logrado llegar a la etapa de madurez por un manejo contable dentro de sus administraciones.

El $40 \%$ de los empresarios ha invertido entre 250000 a 500000 dólares americanos, el $39 \%$ ha optado por $\$ 500000$ a 750000 , el $11 \%$ ha empezado con sus negocios con más de $\$ 1000000$, y el valor más bajo de inversión equivale al 10\%. El $78 \%$ de los inversionistas han accedido a créditos para poder crear sus empresas que actualmente prestan el servicio de alojamiento turístico, mientras que el $22 \%$ han realizado por otros medios.

Se determinó que los dos componentes de prioridad para los empresarios al momento de reinvertir es la Venta - MKT y la infraestructura siendo estos el 57\% y 37\% de la totalidad. Mientras que los componentes de menor prioridad son: tecnología, capacitación-formación y otros, resultado un equivalente individual del $1 \%$ cada uno. El $58 \%$ de los inversionistas cuando invierten esperan que los resultados se evidencien a mediano plazo, mientras que el $24 \%$ desea que sea a corto plazo y el $18 \%$ a largo plazo.

Se evidencia que el $49 \%$ de los huéspedes se encuentran en un rango de 30 a 39 años de edad, el $28 \%$ son de 40 a 49 años, el $18 \%$ tienen de 50 a 59 años y finalmente el rango de menor edad es de 20 a 29 años.

En cuanto a alianzas con agencias de viaje se evidencia que 59 sujetos de estudio tienen un rango mínimo de huéspedes por medio de agencias de viaje, 24 inversionistas han recibido huéspedes un número representativo de clientes.

El 58\% de los inversionistas cuando invierten esperan que los resultados se evidencien a mediano plazo, mientras que el $24 \%$ desea que sea a corto plazo y el $18 \%$ a largo plazo.

2. Componente 2: Procedencia del empresario, Cómo dispone de las utilidades el empresario, Modalidad de viaje del cliente, Plaza del establecimiento (camas), Porcentaje de huéspedes que llegan por medio de Agencias de viajes

Los empresarios nacionales en un $60 \%$ son de procedencia amazónica y $27 \%$ de la región sierra. En el cantón Tena el 13\% de los inversionistas son extranjeros.

Se evidencia que el $99 \%$ de los inversionistas reinvierten en la misma empresa mientras que el $1 \%$ decide invertir en otros temas no relacionados a su lugar de trabajo. Para los prestadores de servicio de alojamiento turístico el $74 \%$ de los clientes llegan a sus establecimientos en familia, mientras que el $13 \%$ en pareja, el $11 \%$ de negocios y el $2 \%$ solos. 
El rango de plazas que tienen los establecimientos de alojamiento es en mayor porcentaje de 1 a 35 plazas mismo que tiene relación con el tipo de establecimientos que se menciona en el componente 1. El 34\% poseen un número de plazas entre 36 a 65 plazas, un 10\% manifiestan que tienen un rango de 66 a 95 plazas y el $8 \%$ manifiesta que tiene más de 96 plazas.

En cuanto a alianzas con agencias de viaje se evidencia que 59 sujetos de estudio tienen un rango mínimo de huéspedes por medio de agencias de viaje, 24 inversionistas han recibido huéspedes un número representativo de clientes.

3. Componente 3: Género del empresario, Etapa de la empresa, Procedencia del cliente, Motivación de viaje del cliente.

Se puede evidenciar que el $53 \%$ de empresarios son de género masculino y el $47 \%$ femenino. El $71 \%$ de las empresas de alojamiento turísticos se encuentran en la etapa de madurez, el $20 \%$ están en crecimiento ya que se han registrado en los últimos tres años y el $10 \%$ en declinación debido a lo cual están presentando los documentos para darse de baja en la entidad reguladora.

Se detalla la frecuencia y porcentaje de las opciones con respuesta según el punto de vista del inversionista. El cliente según los encuestados en un $61 \%$ es nacional mientras que el $34 \%$ de origen extranjero, específicamente europeos, el $89 \%$ de los clientes han llegado a los establecimientos de alojamiento turístico por turismo, el $10 \%$ por motivos de negocios y finalmente el $1 \%$ para visitar a sus familiares.

4. Componente 4: Tipo de habitación que solicita el cliente frecuentemente

Según los empresarios turísticos el tipo de habitación que frecuentemente solicita el cliente es el triple representado con un $45 \%$. Las habitaciones dobles y cuádruples en un $22 \%$ y $23 \%$ respectivamente. Los datos de la Figura 15 tiene relación con la Figura 14 ya que por viajar con grupos grandes de integrantes la necesidad del cliente es el confort y la comodidad al llegar a su destino de viaje.

\section{Componente 5: Profesión del empresario, Edad del empresario}

Se puede apreciar que el $63 \%$ de empresarios poseen una formación académica diferente al ámbito turístico, mientras que el $17 \%$ ha optado por especializarse en ramas afines al turismo. Finalmente, se concluye que el $20 \%$ a pesar de no poseer profesión se dedican a la prestación de servicios turísticos.

El 93\% de los empresarios turísticos se encuentran en un rango mayor a 50 años de edad. Mientras que el 7\% se encuentran en un rango de edad entre los 30 a 49 años.

6. Componente 6: Razón social de la empresa, Factor para toma de decisiones

Los establecimientos turísticos dedicados al servicio de alojamiento trabajan de forma aliada con agencias de viajes locales y otro grupo de forma independiente, esto incide en la tasa de ocupación de la empresa. La gran mayoría ha empezado como emprendimientos 
turísticos y el personal que labora ha empezado de forma empírica. El conocimiento sobre los procesos turísticos en cada área ha sido obtenido mediante la experiencia laboral y con los cursos ofertados por el Ministerio de Turismo.

Los factores según la perspectiva de los empresarios turísticos, donde se evidencia que el $79 \%$ considera que el factor más importante es la competencia al momento de la toma de decisiones. El 12\% atribuye su decisión de inversión al riesgo sobre todo el relacionado al mercado y la competencia, el $4 \%$ considera a la incertidumbre y en menores porcentajes la intervención del gobierno, nivel de ingresos y el tiempo de retorno.

\section{Discusión.}

Los resultados de esta investigación permitieron determinar las características que tiene el servicio de alojamiento turístico que se oferta en el cantón Tena. Adicionalmente se identificó que los factores predominantes para la toma de decisiones, que según la perspectiva de los empresarios turísticos son: competencia, riesgo, incertidumbre, intervención del gobierno, nivel de ingresos y el tiempo. Datos que se pueden evidenciar en la Figura 2.

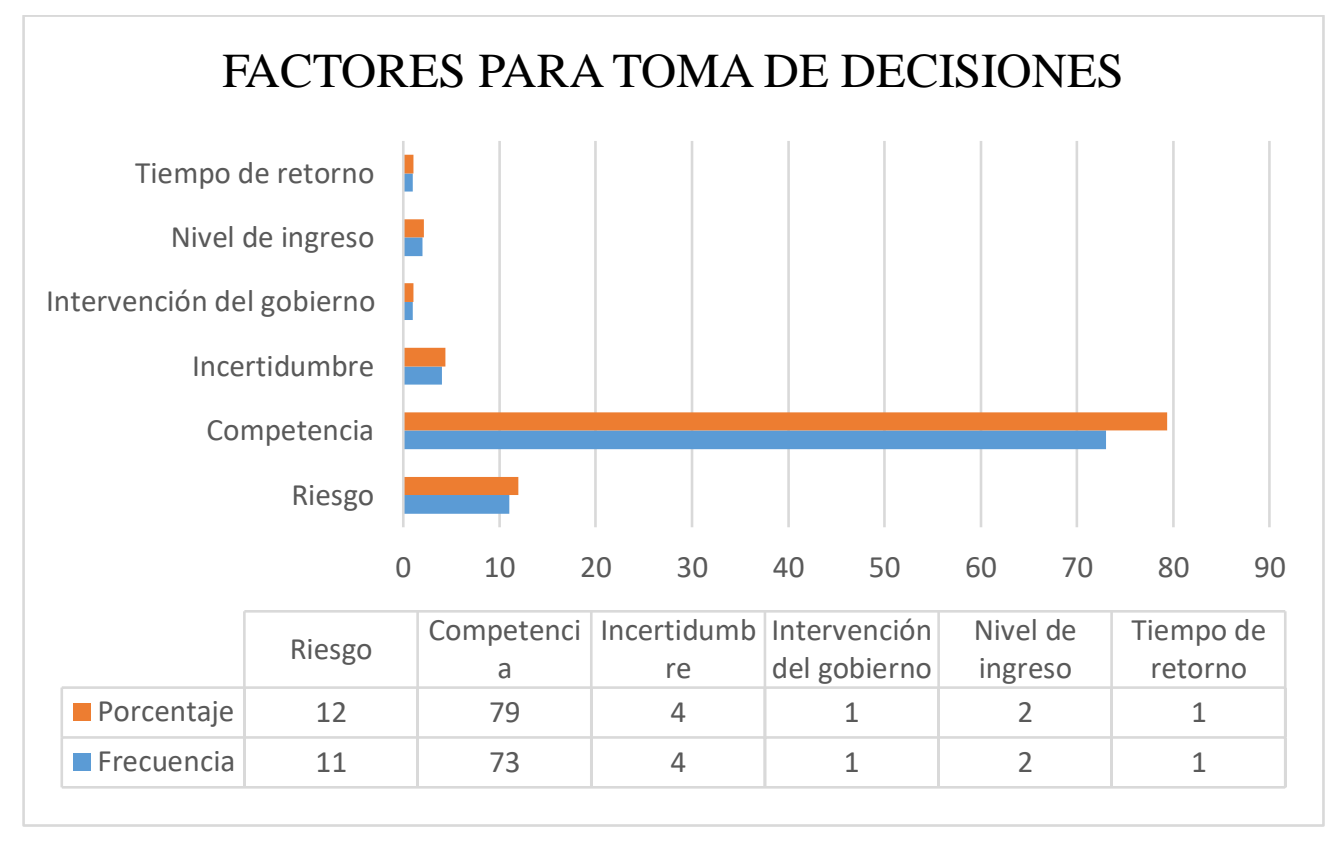

Figura 2. Factores para toma de decisiones

Fuente: Elaboración propio con datos obtenidos con el procesador estadístico computacional, IBM SPSS Statistics 22. Algoritmos Chicago: IBM SPSS Inc.

En el estudio llevado a cabo por Valencia, L. (2018)denominada "Principales Factores Que Determinan La Decisión De Inversión En La Industria Hotelera En La Provincia De Huamanga - Centro Histórico - Año 2017', determina como factor predominante a los beneficios que reciben los prestadores del servicio de hotelería al momento de la toma de decisiones. Así como también al riesgo y la incertidumbre. Además, toma en consideración al riesgo ya sea de carácter socioeconómico como institucional. 
Cada zona de estudio podrá tener similitud en algunos aspectos, pero la realidad en territorio es diferente, es decir, el comportamiento de los inversionistas dependerá según las oportunidades locales, las facilidades financieras y las políticas que el estado establece a favor del desarrollo y crecimiento económico. Es evidente la incidencia de la política pública y financiera que engloba al sector turístico en el cantón Tena.

Actualmente, con la participación de la academia, el sector público y privado se han ejecutado estudios con el objetivo de entender y buscar alternativas ante la pandemia por COVID-19, la Universidad Técnica Particular de Loja publicó el 29 de abril, (2020)un artículo que plasma una visión ante posibles escenarios con datos basados en la Organización Mundial del Turismo, donde señala una posible disminución de ingresos de turistas que varía entre el $20 \%$ y el $30 \%$. Lo que significa que el servicio de alojamiento turístico debe innovar y buscar nuevas estrategias incluyendo el buen manejo y ejecución de las normas de bioseguridad. La oferta debe adaptarse a las necesidades de la demanda con parámetros de seguridad.

\section{Conclusiones.}

- En el cantón Tena, existen establecimientos que ofertan el servicio de alojamiento turísticos consolidados en su mayoría por empresarios donde su formación no está específicamente relacionada al turismo, sino que considerando las ventajas de este territorio han decidido invertir para satisfacer las necesidades de desplazamiento con fines de recreación, negocios y esparcimiento de los potenciales clientes. Existen establecimientos que han logrado alcanzar su etapa de madurez con dominio elemental en contabilidad y aplicación mediana en sus establecimientos.

- Según los datos derivados de esta investigación sobre el conocimiento del sistema turístico se puede evidenciar que su desconocimiento tiene un alto nivel de significancia en relación a las demás variables. Los empresarios turísticos en el cantón Tena han iniciado sin investigaciones previas sus actividades y las han ejecutado de forma empírica.

- La demanda registrada y según la perspectiva de los prestadores y/o administradores de los establecimientos de alojamiento turística es nacional en su gran mayoría y con menor frecuencia europea. La motivación de viaje principal es turismo y seguidamente negocios; su modalidad de viaje suele ser con la familia, laboral e inclusive en pareja. Existe un porcentaje de visitantes que prefieren contratar los servicios de alojamiento con intermediarios.

\section{Referencias bibliográficas.}

Hair, J. F., Black, W. C., Babin, B. J., \& Anderson, R. E. (2016). Multivariate Data Analysis. Boston: Pearson.

Ministerio De Turismo. (2016). Sistema de Informacion Estrategica para el Turismo del Ecuador. Obtenido 
https://siete.turismo.gob.ec/reglamento/REGLAMENTO\%20DE\%20ALOJAMI ENTO\%20TURISTICO.pdf

Ministerio de Turismo. (2018). Obtenido de https://servicios.turismo.gob.ec/turismocifras

Ministerio de Turismo, Oficina Técnica Napo. (ocutbre de 2019). Catastro Turístico provincia de Napo. Tena, Napo, Ecuador.

Organizacion Mundial del Turismo. (08 de Octubre de 2018). Turismo, Comercio y la OMC: Comunicado conjunto de la OMT, la OMC, el ITC y el WTTC, 2018. Ginebra, Suiza. Obtenido de https://www2.unwto.org/es/press-release/2018-1008/turismo-comercio-y-la-omc-comunicado-conjunto-de-la-omt-la-omc-el-itc-yel-

PRO ECUADOR. (2019). Inversiones. Recuperado el 03 de marzo de 2020, de https://www.proecuador.gob.ec/inversiones-4-2/

Secretaría Nacional de Planificación y Desarrollo. (2011). Sistema Nacional de Información. Recuperado el 03 de marzo de 2020, de http://app.sni.gob.ec/snilink/sni/PORTAL_SNI/data_sigad_plus/sigadplusdiagnostico/1560000270001_ PDOT\%20ACT\%202014_DIAGNOSTICO_sigad2\%20sug_senplades1_BD_20 -02-2015_08-58-05.pdf

Universidad Técnica Particular de Loja. (2020). Recuperado el 28 de septiembre de 2020, de https://noticias.utpl.edu.ec/la-pandemia-por-covid-19-puede-afectar-en-un30-a-los-ingresos-del-turismo-nacional

Valencia Olarte, L. A. (2018). Repositorio. Principales Factores que Determinan la Decision de Inversion En La Industria Hotelera En La Provincia de HuamangaCentro Historico - Año 2017. Lima, Perú.

\section{LC Ciencia}




\section{PARA CITAR EL ARTÍCULO INDEXADO.}

Cifuentes Caiza, J. A., Segura Chávez, E. O., Gavilánez Pacheco, O. E., \& Hernández Espinosa, C. del P. (2021). Caracterización del servicio de alojamiento turístico usando métodos multivariados en el cantón Tena, provincia Napo . ConcienciaDigital, 4(2.2), 88102. https://doi.org/10.33262/concienciadigital.v4i2.2.1780

\section{¿Ciencia}

El artículo que se publica es de exclusiva responsabilidad de los autores y no necesariamente reflejan el pensamiento de la Revista Conciencia Digital.

El artículo queda en propiedad de la revista y, por tanto, su publicación parcial y/o total en otro medio tiene que ser autorizado por el director de la Revista Conciencia Digital.

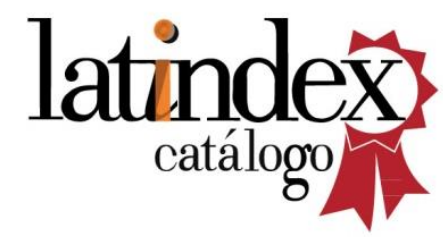

